\title{
Análisis de la calidad y seguridad de la información de aplicaciones móviles en prevención terciaria
}

\section{Javier Martínez-Moreno ${ }^{1,2,3}$, Octaviano Alfredo Martínez-Moreno ${ }^{4}$, Sara Mud-Castelló ${ }^{5}$, Fernando Mud-Castelló ${ }^{5}$, Llanos Moreno Rodriguez ${ }^{6}$, Octaviano Martínez Garví ${ }^{4}$}

1. Servicio de Farmacia. Hospital Universitario Doctor Peset de Valencia (España). 2. Departamento de Farmacia y Tecnología Farmacéutica, Facultad de Farmacia. Valencia (España). 3. Instituto de Reconocimiento Molecular y Desarrollo Tecnológico, Centro Mixto Universidad Politécnica de ValenciaUniversidad de Valencia (España). 4. Farmacéutico comunitario. Madrigueras (Albacete). 5. Farmacéutico comunitario. Ondara (Alicante). 6. Farmacéutico comunitario. Almansa (Albacete).

\section{PALABRAS CLAVE}

Telemedicina, calidad y seguridad de la información, aplicaciones móviles, prevención terciaria

\section{ABREVIATURAS}

TIC: tecnologías de la información y la comunicación SEAIC: Sociedad Española de Alergología e Inmunología Clínica

\section{KEYWORDS}

Telemedicine, quality and data security, mobile applications, tertiary prevention

\section{RESUMEN}

Objetivo: Analizar la calidad y seguridad de la información de las aplicaciones móviles en la App Store de Apple ${ }^{\circledR}$ destinadas a prevención terciaria.

Material y métodos: Estudio observacional transversal de las aplicaciones móviles más populares en la categoría de medicina disponibles en Apple Store ${ }^{\circledR}$ a día de 21 de diciembre de 2014. Las aplicaciones fueron evaluadas con los criterios establecidos en el programa AppSaludable. Se seleccionaron aquellos criterios relacionados con la evaluación de la calidad y seguridad de la información.

Resultados: De las 160 aplicaciones recogidas, sólo 12 aplicaciones estaban incluidas en la prevención terciaria. Todas las aplicaciones recuperadas estaban relacionadas con la adherencia, diabetes, hipertensión y alergia. Las aplicaciones desarrolladas por equipos multidisciplinares fueron las que presentaron mayor rigor en su información,

Conclusiones: La presencia de un profesional sanitario como colaborador de la aplicación parece mejorar la calidad de la información.

Quality and security analysis of mobile applications in tertiary prevention

\section{ABSTRACT}

Objective: Analyze quality and information security of mobile applications in the Apple App Store ${ }^{\circledast}$ used for tertiary prevention.

Methods: Cross-sectional study of the most popular mobile applications in the category of medicine available in Apple Store ${ }^{\circledR}$ to date of 21 December 2014. Criteria established in the AppSaludable program and related to quality and information security criteria were used for applications' assessment.

Results: Of the 160 applications found, only 12 applications were included in tertiary prevention. They were related to adherence, diabetes, hypertension and allergy. Applications developed by multidisciplinary teams showed more rigorous information.

Conclusion: The presence of a health professional as a team member would improve information quality.

Contribución a la autoría: JMM, OAMM, SMC, FMC, LIMR y OMG participaron en el diseño del estudio, el desarrollo de la investigación, el análisis de los resultados, la redacción del manuscrito y en la revisión del contenido final.

Financiación: Ninguna ajena.

Conflicto de intereses: Los autores declaran no existir conflicto de intereses en relación con el contenido del artículo. Cite este artículo como: Martinez-Moreno J, Martinez-Moreno OA, Mud-Castelló S, Mud-Castelló F, Moreno L, Martínez O. Análisis de la calidad y seguridad de la información de aplicaciones móviles en prevención terciaria. Farmacéuticos Comunitarios. 2015 Dec 01; 7(4):23-26. doi:10.5672/FC.2173-9218.(2015/Nol7).004.04 


\section{Introducción}

Las tecnologías de la información y la comunicación (TIC) se han convertido durante los últimos años en una herramienta extraordinaria que ha tomado un papel relevante en la sociedad actual. Las TIC permiten el acceso a una gran cantidad de información y forman parte de una gran variedad de sectores: administración, educación, salud, comercio, empleo, etc.

El término Salud 2.0 (el uso de nuevas tecnologías para la prevención y promoción de la salud) es un nuevo concepto que defiende una visión integral e innovadora del modelo de sanidad requiriendo de nuevos sistemas de información diseñados para interactuar con los profesionales sanitarios, los gestores $\mathrm{y}$, sobre todo, con los ciudadanos/pacientes. En general, se acepta que el término Salud 2.0 (eHealth) fue acuñado por José Cubelos en una tribuna para el diario Expansión en 2007. Este nuevo concepto nos conduce a un enfoque en el cual las TIC serán las herramientas para facilitar el control de la enfermedad y la divulgación de la información sobre estilos de vida saludables que en definitiva conduce a una mayor prevención, adherencia al tratamiento, etc. (1). Este escenario sanitario es el que empezó a desarrollarse durante el siglo xx y que ahora, a principios del siglo xxI, está tomando fuerza. Las aplicaciones móviles son una parte de la telemedicina en constante crecimiento, debido en parte a la gran popularización de los teléfonos móviles con acceso a Internet (smarthphones). Las aplicaciones móviles para la prevención terciaria de la salud se suman a las tradicionales visitas con los profesionales sanitarios y a las campañas sanitarias. Estas aplicaciones serán nuestro objeto de trabajo, debido a su relevancia dentro de la salud pública.

La rápida evolución del software y la proliferación descontrolada, provoca la existencia de numerosas aplicaciones en Google Play ${ }^{\circledR}$ y en App Store de Apple ${ }^{\circledR}$ dando lugar a la fragmentación de la información y a la dificultad para encontrar la aplicación adecuada (2). Además, la falta de validación por profesionales sanitarios cualificados, hace que el contenido en ciertos casos tenga una validez dudosa. Actualmente sólo el 10\% de las aplicaciones son recomendadas por el médico (3). Esta afirmación nos señala que el 90\% de las aplicaciones referentes a la salud son elegidas por la población con el fin de aumentar el conocimiento sobre su patología y de esta forma un mejor control.

Por este motivo se ha creído conveniente analizar la calidad y seguridad de la información de las aplicaciones móviles disponibles más populares en la categoría de medicina dentro de la App Store de Apple ${ }^{\circledR}$ destinadas a prevención terciaria de enfermedad.

\section{Material y métodos}

Estudio observacional transversal de las aplicaciones móviles disponibles en App Store de Apple ${ }^{\circledR}$ a día de 21 de diciembre de 2014. Para obtener el listado de aplicaciones que se evalúan accedimos a las aplicaciones bajo la categoría de "Medicina", la cual muestra las aplicaciones más populares, y escogimos aquellas que tenían la finalidad de prevención terciaria.

Para el análisis ad hoc, se valoraron según los criterios que se muestran en la (figura 1). Dichos criterios han sido obtenidos de un programa iniciado en Andalucía (España) (4), que tiene el fin de garantizar que los procesos y desarrollo de las aplicaciones móviles orientadas a la salud contribuyan a la mejora de los servicios y no al perjuicio de los usuarios. De todos los criterios disponibles se escogieron los relacionados con la calidad y seguridad de la información.

\section{Resultados}

Se recuperaron un total de 160 aplicaciones, de las cuales sólo 12 tenían la finalidad de prevención terciaria, las cuales pueden dividirse en cuatro grupos (figura 2).

La tabla 1 muestra la matriz de resultados de las aplicaciones evaluadas con los criterios establecidos. La aplicación mejor valorada fue Social Diabetes ${ }^{\circledR}$, y las peores valoradas Med reminder $^{\circledR}$, Diabetes app lite ${ }^{\circledR}$ y Pastimed $^{\circledR}$.

Las dos aplicaciones que tienen por objeto pacientes alérgicos están realizadas por la Sociedad Española de Alergología e Inmunología Clínica (SEAIC) y el contenido es totalmente fiable. Referente a las aplicaciones relacionadas con la adherencia al tratamiento, tres de las cuatro que se recuperaron son aplicaciones de muy baja calidad. En cambio hay una apli-
La app de salud se adapta al tipo de destinatarios al que se dirige

La app de salud ofrece información transparente sobre la identidad y localización de sus propietarios.

La app de salud proporciona información sobre sus fuentes de financiación, promoción y patrocinio, asi como posibles conflictos de intereses.

La app de salud identifica a los autores/responsables de sus contenidos, así como su cualificación profesional.

La app de salud advierte de aquellas actualizaciones que inciden o modifican funcionamientos o contenidos sobre salud o cualquier otro dato sensible.

La app de salud está basada en una o más fuentes de información fiable y toma en consideración la evidencia cientifica disponible.

La app de salud proporciona información concisa acerca del procedimiento utilizado para seleccionar sus contenidos.

Figura 1 Criterios evaluados en las diferentes aplicaciones (según escala 0, 1 o 2 según si cumplían el criterio siempre, a veces o nunca respectivamente)

cación, Patient IO, la cual fue una de las más completas en la revisión, ya que detrás existe un trabajo multidisciplinar que ofrece una buena calidad del contenido. Las dos aplicaciones referentes a la monitorización de presión arterial ofrecían contenidos muy similares, es decir, la posibilidad de llevar un registro de tensión arterial en el dispositivo. Por último, las aplicaciones con relación a diabetes, fueron diversas, desde la aplicación Sanofi Diabetes Manager $^{\circledR}$, que no

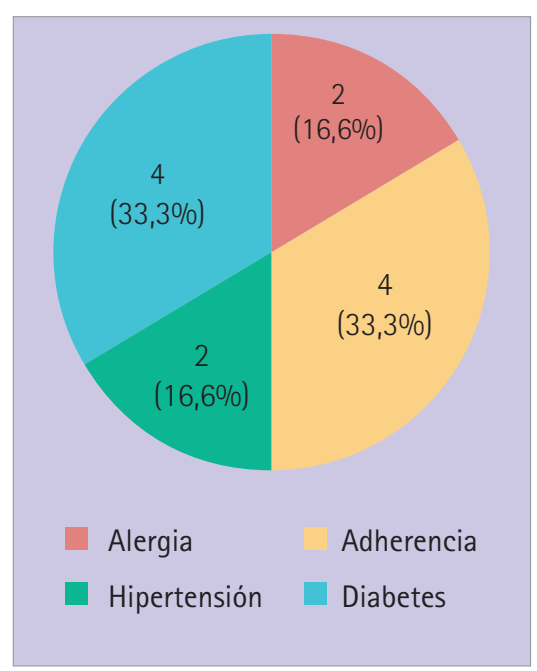

Figura 2 Categorias de aplicaciones filtradas 
funcionaba desde hace un tiempo, hasta Social Diabetes ${ }^{\circledR}$, la cual fue muy completa ya que forma parte de un proyecto liderado por un equipo multidisciplinar.

\section{Discusión}

Las aplicaciones con finalidad de prevención terciaria relacionadas con la adherencia y diabetes seguidas de hipertensión y alergia, son las que más interesan a los usuarios. Esto resulta lógico, puesto que las aplicaciones más buscadas son las relacionadas con las enfermedades más prevalentes en España (5).

El presente trabajo sugiere que aquellas aplicaciones desarrolladas por equipos multidisciplinares son las que contienen mayor calidad y seguridad de la información. Durante nuestro análisis hemos podido comprobar que aquellas en las que están involucrados profesionales sanitarios tienen un contenido más riguroso desde el punto de vista científico. Arnhold M y col. concluyen en su revisión de aplicaciones para la monitorización de la diabetes que deberían de estar involucrados profesionales sanitarios (6) en el desarrollo de éstas con el fin de asegurar una información rigurosa, al tiempo que expertos en desarrollo de software. Wallace LS y col. revisaron aplicaciones disponibles para el autocontrol del dolor, llegando a la conclusión de que la gran mayoría carecía de evidencia suficiente ya que no estaban desarrolladas por profesionales sanitarios cualificados (7). Bender JL y col. revisaron aplicaciones disponibles para el autocuidado de aquellas personas con algún tipo de neoplasia y, al igual que en el estudio anterior, también concluyen con la falta de evidencia que existe en este tipo de aplicaciones móviles (8).

La gran limitación del estudio es la imposibilidad de evaluar la totalidad de aplicaciones referentes a prevención terciaria, debido por una parte al gran número existente, y por otra parte a la nula posibilidad de filtrar dentro de una categoría. Es por ello que el estudio se basa en aquellas más populares relacionadas con la medicina.

El uso de las aplicaciones para la gestión de la información en el paciente enfermo parece constituir de aquí en adelante un recurso indispensable para el intercambio de in- formación, desarrollo de habilidades personales y cambios de estilo de vida, los cuales pueden traducirse en un cambio de paradigma en la reducción de las tasas de morbimortalidad de las enfermedades crónicas (2). En España, más del 45\% de la población mayor de 16 años padece un proceso crónico en el que el grado de adherencia al tratamiento se sitúa en un $50 \%(9,10)$. Para mejorar la adherencia el paciente debe conocer la enfermedad, simplificar en la medida de lo posible el tratamiento y conocer su medicación.

La autogestión y la monitorización remota de pacientes se están convirtiendo en soluciones viables para el manejo de las enfermedades crónicas y los teléfonos inteligentes están jugando un papel muy importante (11-13). Es en este contexto donde la calidad y seguridad de la información juegan un papel muy importante. Para el usuario no experto la selección de una aplicación relacionada con la salud implica tanto dificultades a la hora de identificar información de calidad como riesgos en el acceso de información de poca calidad, sesgada o errónea. Es por ello que la calidad y seguridad de la

Tabla 1 Resultados de la valoración de las aplicaciones

\begin{tabular}{|c|c|c|c|c|c|c|c|c|c|c|c|c|}
\hline Aplicación & 1 & 2 & 3 & 4 & 5 & 6 & 7 & 8 & 9 & 10 & 11 & 12 \\
\hline 1. La app de salud se adapta al tipo de destinatarios al que se dirige. & 1 & 1 & 2 & 2 & 2 & 2 & 2 & 2 & 2 & 2 & 2 & 2 \\
\hline $\begin{array}{l}\text { 2. La app de salud ofrece información transparente sobre la identi- } \\
\text { dad y localización de sus propietarios. }\end{array}$ & 2 & 2 & 0 & 2 & 1 & 2 & 2 & 1 & 0 & 0 & 2 & 2 \\
\hline $\begin{array}{l}\text { 3. La app de salud proporciona información sobre sus fuentes de } \\
\text { financiación, promoción y patrocinio, asi como posibles conflic- } \\
\text { tos de intereses. }\end{array}$ & 2 & 1 & 0 & 0 & 0 & 2 & 2 & 0 & 0 & 0 & 1 & 2 \\
\hline $\begin{array}{l}\text { 4. La app de salud identifica a los autores/responsables de sus con- } \\
\text { tenidos, asi como su cualificación profesional. }\end{array}$ & 2 & 2 & 0 & 0 & 0 & 2 & 2 & 0 & 0 & 0 & 2 & 2 \\
\hline $\begin{array}{l}\text { 5. La app de salud advierte de aquellas actualizaciones que inciden } \\
\text { o modifican funcionamientos o contenidos sobre salud o cual- } \\
\text { quier otro dato sensible. }\end{array}$ & 2 & 2 & 2 & 2 & 2 & 2 & 0 & 2 & 2 & 2 & 2 & 1 \\
\hline $\begin{array}{l}\text { 6. La app de salud está basada en una o más fuentes de infor- } \\
\text { mación fiable y toma en consideración la evidencia científica } \\
\text { disponible. }\end{array}$ & 2 & 2 & 0 & 0 & 0 & 2 & 2 & 0 & 2 & 1 & 2 & 2 \\
\hline $\begin{array}{l}\text { 7. La app de salud proporciona información concisa acerca del pro- } \\
\text { cedimiento utilizado para seleccionar sus contenidos. }\end{array}$ & 2 & 2 & 0 & 2 & 2 & 2 & 2 & 0 & 0 & 0 & 2 & 2 \\
\hline TOTAL & 13 & 12 & 4 & 8 & 7 & 14 & 12 & 5 & 6 & 5 & 13 & 13 \\
\hline
\end{tabular}

1 = Alergo alarm, 2 = Polenes.com mobile, $3=$ Med reminder, $4=$ Tensión arterial, $5=$ Presión arterial monitor, $6=$ Social Diabetes, $7=$ Sanofi diabetes manager, 8 = Diabetes app lite, $9=$ Alarma de medicamento, 10 = Pastimed, $11=$ Glucose Buddy y $12=$ Patient 10 . 
información debe ser una característica inherente en toda aplicación móvil con contenido sanitario. La presencia de un profesional sanitario en su desarrollo y seguimiento, o la evaluación externa por parte de sanitarios se hace totalmente necesaria. En este sentido, diversas entidades han elaborado sellos de calidad, códigos de conducta, acreditaciones o recomendaciones con el fin de informar y asegurar a los pacientes que se respetan unos mínimos de calidad.

\section{Conclusiones}

Las aplicaciones desarrolladas por equipos multidisciplinares son las más rigurosas en la calidad y seguridad de la información. Por lo tanto, la presencia de un profesional sanitario como colaborador de la aplicación parece mejorar la calidad de la información.

\section{Referencias bibliográficas}

1. Fernández M. Impacto de la Web 2.0 (redes sociales) en la información sobre salud [Internet]. En: Sistema Económico Latinoamericano y del Caribe (SELA), editores. Informe Final del II Seminario sobre Telemedicina y Salud-e: Prácticas de Innovación y Estándares; 2011 oct 19-21; Caracas, Venezuela. Caracas: SELA; 2011. (SP/II-SR-SeTALC:Pie/ Di No 29-11). [Acceso 26/12/2014] Disponible en: http://www.sela.org/ attach/258/default/Impacto_de_la_ Web_2.0_y_las_Redes_Sociales_ en_lainformacion_sobre_salud.pdf 2. Van Velsen L, Beaujean DJ, van Gemert-Pijnen JE. Why mobile health app overload drives us crazy, and how to restore the sanity. BMC Medical Informatics and Decision Making. 2013;13:23. doi:10.1186/1472-694713-23

3. Bauer AM, Rue T, Keppel GA, Cole AM, Baldwin L-M, Katon W. Use of Mobile Health (mHealth) Tools by Primary Care Patients in the WWAMI Region Practice and Research Network (WPRN). J Am Board Fam Med [Internet]. 2014 11-1;27(6):780-8. [Acceso 26/12/2014]. Disponible en: http://www.jabfm.org/content/27/6/780

4. Estrategia de calidad y seguridad en aplicaciones móviles de salud - Calidad y seguridad de la información [Internet]. [Acceso 15/1/2015]. Disponible en: http://www.calidadappsalud.com/recomendaciones/calidad-seguridad-informacion/

5. Instituto Nacional de Estadística [Internet] Encuesta Nacional de Salud 2011-2012 (2013) Cifras INE. [Acceso 15/1/2015]. Disponible en: www.ine.es

6. Arnhold M, Quade M, Kirch W. Mobile Applications for Diabetics: A Systematic Review and Expert-Based Usability Evaluation Considering the Special Requirements of Diabetes Patients Age 50 Years or Older. Eysenbach G, ed. Journal of Medical Internet Research. 2014;16(4):e104. doi:10.2196/jmir.2968.

7. Wallace LS, Dhingra LK. A systematic review of smartphone applications for chronic pain available for download in the United States. J Opioid Manag. 2014;10(1):63-68. doi:10.5055/jom. 2014.0193
8. Bender JL, Yue RYK, To MJ, Deacken L, Jadad AR. A Lot of Action, But Not in the Right Direction: Systematic Review and Content Analysis of Smartphone Applications for the Prevention, Detection, and Management of Cancer. Potts H, ed. Journal of Medical Internet Research. 2013;15(12):e287. doi:10.2196/jmir.2661

9. Estrategia de promoción de la salud y prevención en el SNS. Madrid: Ministerio de Sanidad, Servicios Sociales e Igualdad; 2012.

10. Conthe $\mathrm{P}$, Márquez Contreras E, Aliaga Pérez A, Barragán García B, Fernández de Cano Martín MN, González Jurado M, et al. Adherencia terapéutica en la enfermedad crónica: estado de la situación y perspectiva de futuro. Revista Clínica Española [Internet]. 2014 Aug;214(6):336-44. [Acceso 15/1/2015]. Disponible en: http:// www.sciencedirect.com/science/article/pii/S0014256514001271

11. Marshall A, Medvedev 0, Antonov A. Use of a Smartphone for Improved Self-Management of Pulmonary Rehabilitation. International Journal of Telemedicine and Applications. 2008, Article ID 753064, 5 pages. doi:10.1155/2008/753064

12. Hong S, Kim S, Kim J, Lim D, Jung S, Kim D, et al: Portable emergency telemedicine system over wireless broadband and 3G networks. [31st Annual International Conference of the IEEE EMBS]. Minneapolis, Minnesota, USA: IEEE; 2009:12501253.

13. Krishna S, Boren SA, Balas EA. Telemedicine and e-Health. April 2009;15(3):231-240. doi:10.1089/tmj. 2008.0099. 